\title{
Acid-base balance, serum electrolytes and need for non-invasive ventilation in patients with hypercapnic acute exacerbation of chronic obstructive pulmonary disease admitted to an internal medicine ward
}

\author{
Alfonso Schiavo ${ }^{1}$, Maurizio Renis ${ }^{1}$, Mario Polverino ${ }^{2 *}$, Arcangelo lannuzzi ${ }^{3}$ and Francesca Polverino ${ }^{4}$
}

\begin{abstract}
Background: Hypoventilation produces or worsens respiratory acidosis in patients with hypercapnia due to acute exacerbations of chronic obstructive pulmonary disease (AECOPD). In these patients acid-base and hydroelectrolite balance are closely related. Aim of the present study was to evaluate acid-base and hydroelectrolite alterations in these subjects and the effect of non-invasive ventilation and pharmacological treatment.

Methods: We retrospectively analysed 110 patients consecutively admitted to the Internal Medicine ward of Cava de' Tirreni Hospital for acute exacerbation of hypercapnic chronic obstructive pulmonary disease. On admission all patients received oxygen with a Venturi mask to maintain arterial oxygen saturation at least $>90 \%$, and received appropriate pharmacological treatment. Non-Invasive Ventilation (NIV) was started when, despite optimal therapy, patients had severe dyspnea, increased work of breathing and respiratory acidosis. Based on Arterial Blood Gas $(A B G)$ data, we divided the 110 patients in 3 groups: $A=51$ patients with compensated respiratory acidosis; $B=36$ patients with respiratory acidosis + metabolic alkalosis; and $C=23$ patients with respiratory acidosis + metabolic acidosis. 55 patients received only conventional therapy and 55 had conventional therapy plus NIV.

Results: The use of NIV support was lower in the patients belonging to group B than in those belonging to group A and C ( $25 \%$, vs $47 \%$ and $96 \%$ respectively; $p<0.01)$. A statistically significant association was found between $\mathrm{PCO}_{2}$ values and serum chloride concentrations both in the entire cohort and in the three separate groups.

Conclusions: Our study shows that in hypercapnic respiratory acidosis due to AECOPD, differently from previous studies, the metabolic alkalosis is not a negative prognostic factor neither determines greater NIV support need, whereas the metabolic acidosis in addition to respiratory acidosis is an unfavourable element, since it determines an increased need of NIV and invasive mechanical ventilation support.
\end{abstract}

Keywords: COPD, Acid-base balance, Hypercapnia, NIV

\footnotetext{
* Correspondence: polverinomario@alice.it

${ }^{2}$ Division of Pulmonary Medicine, Scafati Hospital, Salerno, Italy

Full list of author information is available at the end of the article
} 


\section{Background}

Hypercapnic respiratory failure is a complex clinical and functional condition, characterized by an alteration of the acid/base $(\mathrm{AB})$ balance, associated with multi-organ impaired function. Several physiological systems are involved in the control of the $\mathrm{AB}$ balance, namely the respiratory system, the kidney, as well as red blood cells and blood proteins, and the bicarbonate buffering system. The $\mathrm{AB}$ balance and hydro-electrolytic ( $\mathrm{HE}$ ) balance are closely related, as for any increase in $\mathrm{CO}_{2}$ (respiratory acidaemia), a counterbalancing metabolic alkalosis occurs as main compensatory mechanism achieved by a complex ion urinary excretion mechanism [1].

Therefore, in order to manage the $\mathrm{AB}$ disorders it is necessary to understand its fine regulatory mechanisms.

For the interpretation of $\mathrm{AB}$ disorders two approaches can be used: a physical-chemical approach (which relies on the theory of the Strong Ion Difference [SID]) or a pathophysiologic approach (which relies on the compensation laws). Many authors consider the physicochemical approach a complex and unfeasible approach, and poorly matching with the clinical reality. Moreover, it requires many factors to calculate the SID. On the other hand, the physio-pathological approach is easier and much more reliable, because it provides a quantitative measurement of the $\mathrm{AB}$ compensatory responses. Many trials, performed in different clinical disorders, have supported its use in humans [2].

In this study we used the pathophysiologic approach, according to the compensation laws, to evaluate the Chronic Obstructive Pulmonary Disease (COPD) acute exacerbations.

In hypercapnic AECOPD the hypoventilation produces or worsens respiratory acidosis. Since most of these patients, especially if elderly and critical, are multi-drugs recipients for comorbidities, the $\mathrm{AB}$ and $\mathrm{HE}$ disorders are very common, producing a potential bias in the interpretation of the final values [3].

Thus, we sought to evaluate: 1 ) the $\mathrm{AB}$ and $\mathrm{HE}$ disorders in hypercapnic AECOPD patients; and 2) the effect of the type of treatment (pharmacological or by noninvasive ventilation $[\mathrm{NIV}]$ ) on the $\mathrm{AB}$ and $\mathrm{HE}$ disorders,

\section{Methods}

We retrospectively analysed the data of inpatients admitted in 2012 to the Internal Medicine Division of Cava de' Tirreni Hospital (Italy) for hypercapnic AECOPD.

COPD and AECOPD were defined according to the GOLD guidelines [4]. Hypercapnia was defined as $\mathrm{PaCO}_{2}>$ $45 \mathrm{mmHg}$ [4].

Between January and December 2012, 140 patients were admitted for hypercapnic AECOPD. Thirty patients were excluded for the following reasons: 10 patients were on NIV home care, 7 had a pneumonia, 3 had
ARDS, 10 had severe respiratory failure $(\mathrm{pH}<7.1$, hypercapnic coma or hemodynamic instability) and were admitted to the Intensive Care Unit (ICU). Information about comorbidities were obtained from the primary care physicians.

Anthropometric characteristics, clinical parameters, haematological and biochemical exams, and blood gas analyses were assessed upon admission. The Glasgow Coma Scale was used for the state of consciousness [5].

Patients were classified into three categories: $\mathrm{A}=\mathrm{com}$ pensated respiratory acidosis; $\mathrm{B}=$ respiratory acidosis with metabolic alkalosis; and $\mathrm{C}=$ respiratory acidosis with metabolic acidosis [6].

On admission all patients received oxygen with a Venturi mask to maintain arterial oxygen saturation $\left(\mathrm{SaO}_{2}\right)$ at least $>90 \%$, and received treatment, besides the treatments for the comorbidities, with conventional therapy: nebulized bronchodilators, systemic glucocorticoids, and antibiotics [7].

NIV was started when the oxygen therapy and optimal pharmacological treatment did not produce any clinical improvement and patients had severe dyspnea, increased work of breathing and respiratory acidosis $(\mathrm{pH}<7.35$ or arterial carbon dioxide pressure $>45 \mathrm{mmHg}$ or both). All patients were conscious and able to protect their airway and deal with respiratory secretions.

NIV was performed through oronasal mask with a pressure/volume ventilator (Carat II, Hoffrichter). Support pressure, Positive End Expiratory Pressure (PEEP) and triggered flows were adjusted to obtain a tidal volume of $6-8 \mathrm{ml} / \mathrm{kg}$, the best possible oxygenation, and a decrease in respiratory rate. Support pressure and PEEP were modified based on the arterial gases. The mean Inspiratory Positive Assisted Pressure (IPAP) was $16 \pm 4$ $\mathrm{cmH}_{2} \mathrm{O}$. None of the patients refused the treatment or required discontinuation of ventilation for discomfort, NIV was suspended when clinical steady-state was achieved: respiratory rate $<24 / \mathrm{min}$, heart rate $<110 /$ beats-min, $\mathrm{pH}>7.35$ and $\mathrm{SaO}_{2}>90 \%$ with $\mathrm{FiO}_{2}<40 \%$ [8].

Patients were transferred to ICU for invasive mechanical ventilation if the following conditions occurred: 1) worsening of arterial $\mathrm{pH}$ and $\mathrm{PaCO}_{2} ; 2$ ) clinical signs of pre-coma; 3) cardiovascular instability [9].

Based on ABG data, according to the compensation laws, we divided the 110 patients in the 3 groups previously identified: $A=51$ patients. with compensated respiratory acidosis; $\mathrm{B}=36$ patients with respiratory acidosis + metabolic alkalosis; and $\mathrm{C}=23$ patients. with respiratory acidosis + metabolic acidosis.

\section{Statistical analysis}

All quantitative variables are reported as mean \pm standard deviation (SD). 
Statistical analyses were conducted using parametric or nonparametric tests, based on the continuous or asymmetrical distribution of the variables. Student's $T$ test for unpaired data was used to compare the quantitative variables between the two groups: patients in NIV support $(\mathrm{NIV}+)$ vs patients treated with conventional therapy (NIV-). The $\chi^{2}$ test was used to compare ordinal variables.

The odds ratio (OR) for NIV+ vs NIV- and the $95 \%$ confidence interval $(95 \% \mathrm{CI})$ were calculated using a logistic regression analysis for the 3 categories of ABG disorders, and group B (respiratory acidosis + metabolic alkalosis), which showed the lower percentage of NIV support, was used as reference group.

Linear regression analysis was used to evaluate the association between $\mathrm{pCO}_{2}$ and blood electrolytes.

The statistical analysis was performed using Statistical Package for Social Science (SPSS 12).

\section{Results}

The patients with COPD and hypercapnia had the following comorbidites: chronic ischaemic heart disease (35\%); atrial fibrillation (15\%); heart failure (32\%); previous stroke (11\%); diabetes mellitus (26\%); chronic renal failure $(44 \%)$. Twenty-eight percent of patients had peripheral edema.

The vast majority of patients was receiving cardiovascular therapy: diuretics (86\%), potassium-sparing diuretics (6\%), ACE inhibitors (25\%), angiotensin receptor antagonists (29\%), digoxin (9\%), selective beta blockers (6\%), calcium channel blockers (21\%), nitro-derivatives (10\%), as well as antidiabetic medications, aspirin, statins and inhaled bronchodilators.

Fifty-five subjects required NIV. In 33 patients out of the 55 , the acidosis was corrected by the NIV, whereas the remaining 22, in which the acidosis persisted, were transferred to ICU. The mean NIV time, in successfully treated patients, was $7.8 \pm 5.0$ days.

\section{NIV+ vs NIV-}

Anthropometric data, clinical, biochemical and ABG parameters are shown in Table 1 . Patients were divided into two groups: standard therapy (NIV -) and standard therapy plus NIV (NIV +).

Compared to NIV -, patients of NIV + had, in addition to higher $\mathrm{PaCO}_{2}$ and lower values of $\mathrm{pH}$ and $\mathrm{PaO}_{2} / \mathrm{FiO}_{2}$, increased heart rate, higher values of blood potassium, bicarbonates and lactates and lower albumin and serum chloride concentrations (Table 1).

\section{NIV and ABG groups}

The use of NIV support was lower in the patients belonging to group B (respiratory acidosis + metabolic alkalosis) than those belonging to group A (compensated
Table 1 Clinical and metabolic parameters (mean \pm SD)

\begin{tabular}{|c|c|c|c|}
\hline Variables & $N I V-(N=55)$ & $N I V+(N=55)$ & $p$ \\
\hline Age (years) & $77.5 \pm 8.8$ & $77.6 \pm 10.7$ & 0.94 \\
\hline Gender (M/F) & $29 / 26$ & $28 / 27$ & 0.85 \\
\hline Glucose (mg/dl) & $142.3 \pm 48.9$ & $156.2 \pm 76.5$ & 0.26 \\
\hline Serum Uric Acid (mg/dl) & $6.3 \pm 2.0$ & $6.6 \pm 2.6$ & 0.60 \\
\hline Serum Albumin (g/dl) & $3.3 \pm 0.5$ & $2.9 \pm 0.5$ & $<0.001$ \\
\hline C-Reactive-Protein (mg/l) & $76.9 \pm 75.7$ & $27.4 \pm 34.4$ & 0.039 \\
\hline Serum Creatinine (mg/dl) & $1.08 \pm 0.58$ & $1.29 \pm 0.88$ & 0.14 \\
\hline GFR (MDRD ml/min/1.73 $\mathrm{m}^{2}$ ) & $71.8 \pm 32.0$ & $72.7 \pm 49.3$ & 0.91 \\
\hline HR (bpm) & $83.8 \pm 12.7$ & $93.6 \pm 13.6$ & $<0.001$ \\
\hline SBP $(\mathrm{mmHg})$ & $124.1 \pm 17.0$ & $120.0 \pm 17.9$ & 0.21 \\
\hline $\mathrm{DBP}(\mathrm{mmHg})$ & $74.2 \pm 9.9$ & $72.5 \pm 10.2$ & 0.38 \\
\hline $\mathrm{PaO}_{2}(\mathrm{mmHg})$ & $58.4 \pm 13.5$ & $60.2 \pm 16.2$ & 0.54 \\
\hline $\mathrm{PaO}_{2} / \mathrm{FiO} 2$ & $255.9 \pm 38.6$ & $200.2 \pm 57.6$ & $<0.001$ \\
\hline $\mathrm{pH}$ & $7.42 \pm 0.05$ & $7.29 \pm 0.11$ & $<0.001$ \\
\hline $\mathrm{PaCO}_{2}(\mathrm{mmHg})$ & $50.5 \pm 5.5$ & $75.5 \pm 19.8$ & $<0.001$ \\
\hline Lactate (mmol/L) & $1.38 \pm 0.68$ & $2.01 \pm 1.76$ & 0.017 \\
\hline Serum $\mathrm{HCO}^{-}$(mmol/L) & $32.6 \pm 5.5$ & $36.0 \pm 10.1$ & 0.036 \\
\hline Serum $\mathrm{Na}^{+}(\mathrm{mmol} / \mathrm{L})$ & $138.6 \pm 3.9$ & $139.1 \pm 6.9$ & 0.63 \\
\hline Serum K+ $(\mathrm{mmol} / \mathrm{L})$ & $4.4 \pm 0.6$ & $4.7 \pm 0.8$ & 0.037 \\
\hline Serum $\mathrm{Ca}^{++}(\mathrm{mmol} / \mathrm{L})$ & $8.6 \pm 0.6$ & $8.4 \pm 0.8$ & 0.12 \\
\hline Serum $\mathrm{Mg}^{+}(\mathrm{mmol} / \mathrm{L})$ & $1.96 \pm 0.32$ & $1.89 \pm 0.32$ & 0.24 \\
\hline Serum $\mathrm{Cl}^{-}(\mathrm{mmol} / \mathrm{L})$ & $99.4 \pm 3.9$ & $96.6 \pm 8.1$ & 0.030 \\
\hline
\end{tabular}

GFR Glomerular Filtration Rate, MDRD Modification of Diet in Renal Disease

respiratory acidosis) and group $\mathrm{C}$ (respiratory acidosis + metabolic acidosis, $25 \%$, vs $47 \%$ and $96 \%$ respectively; $p<0.01$ ) (Table 2).

Using a logistic regression model, we observed that the probability of requiring NIV, in comparison to the reference group $\mathrm{B}$, was approximately 3 times higher in patients of group A (OR 2.7; $95 \%$ CI 1.04-6.82; p <0.05) and 67 times higher in patients of group C (OR 67.1; $95 \%$ CI 7.9-572.8; $p<0.001)$.

\section{Electrolytes, edema and medications in the 3 groups}

In a variance analysis, a statistically significant difference in serum albumin concentrations was found in group $C$ $(2.7 \pm 0.11 \mathrm{~g} / \mathrm{dL})$ compared to group B $(3.2 \pm 0.09 \mathrm{~g} / \mathrm{dL}$; $p=0.012)$ and group A $(3.2 \pm 0.07 \mathrm{~g} / \mathrm{dL} ; p=0.003)$.

Significant hyperlactatemia (lactate values $\geq 2 \mathrm{mmol} / \mathrm{L}$ ) was found in all groups, even though in different percentages: in group B, patients with symptomatic hyperlactatemia were all in conventional therapy (6/ 20: $33 \%$ of subjects in NIV- and no one in NIV +); in the group A: $4 / 20$ (25 \%) in NIV - and 6/18 (33.3 \%) in NIV +, and in the group C: $11 / 22$ (50 \%) in NIV +, and no one in NIV -. 
Table 2 Non-Invasive-Ventilation (NIV +) in groups of patients according to ABG analysis values

\begin{tabular}{lcrr}
\hline & \multicolumn{2}{l}{ Mixed respiratory acidosis - metabolic } & \multicolumn{2}{c}{$\begin{array}{l}\text { Compensated respiratory } \\
\text { acidosis }(N=51)\end{array}$} & \multicolumn{2}{l}{$\begin{array}{l}\text { Mixed respiratory - metabolic } \\
\text { acidosis }(N=23)\end{array}$} \\
\hline NIV - & $75 \%(27)$ & $53 \%(27)$ & $4 \%(1)$ \\
NIV + & $25 \%(9)$ & $47 \%(24)$ & $96 \%(22)$ \\
\hline
\end{tabular}

$x^{2}<0.001$

The incidence of peripheral edema was not statistically different between the 3 ABG groups: $25 \%$ in group B, $26 \%$ in group A, and $47 \%$ in group C.

A statistically significant association was found between $\mathrm{pCO}_{2}$ values and serum chloride concentrations both in the entire cohort and in the three separate groups (Table 3 ).

Table 4 shows the list of medication taken for cardiovascular conditions.

\section{Discussion}

The hypercapnic AECOPD is an alarming event that requires a careful management. Several factors determine the final outcome in terms of both survival and need for assisted mechanical ventilation in ICU. These factors are patient's age, general health status, disease severity, hemodynamic stability, concomitant comorbidities, pulmonary function, respiratory acidosis degree, and underlying $\mathrm{AB}$ and $\mathrm{HE}$ balance disorders [10].

This is a retrospective study, in which patients with hypercapnic AECOPD, managed in an internal medicine ward of a General Hospital have been studied. We have shown that the group of patients with respiratory acidosis + metabolic acidosis (group C) had the most serious prognosis, both in terms of non-invasive ventilation, and ICU support. These patients often underestimate their clinical status so that an acute event easily leads to metabolic derangement, most of the times caused by renal failure or worsening of diabetes mellitus. However, in some patients, adequate medical treatment of the metabolic imbalance, and the correction of hypoxemia and hypercapnia by oxygen and NIV could allow a safe management with quick recovery. In patients with hypercapnic AECOPD, a good metabolic compensation and an adequate renal function significantly reduce the mortality [11].

In respiratory acidosis, if an excessive increase of bicarbonate concentration (higher than expected by renal compensation laws) occurs, a mixed disorder with autonomous metabolic alkalosis should be suspected. In patients with multiple comorbidities and multi-drug treatment, several circumstances could lead to metabolic

Table 3 Linear association among $\mathrm{pCO}_{2}$ and serum electrolytes in groups of patients according to ABG analysis values and in the entire cohort

\begin{tabular}{|c|c|c|c|c|c|}
\hline Variables & & $\begin{array}{l}\text { Mixed respiratory acidosis - } \\
\text { metabolic alkalosis }(N=36)\end{array}$ & $\begin{array}{l}\text { Compensated respiratory } \\
\text { acidosis }(N=51)\end{array}$ & $\begin{array}{l}\text { Mixed respiratory - metabolic } \\
\text { acidosis }(N=23)\end{array}$ & $\begin{array}{l}\text { Hypercapnic COPD, } \\
\text { entire group }(N=110)\end{array}$ \\
\hline \multirow[t]{3}{*}{$\overline{\mathrm{Na}^{+}}$} & $R^{2}$ & 0.10 & 0.01 & 0.16 & 0.002 \\
\hline & Slope & 0.67 & 1.06 & -1.33 & -0.02 \\
\hline & $\mathrm{p}$ & 0.06 & 0.03 & 0.06 & 0,62 \\
\hline \multirow[t]{3}{*}{$\mathrm{K}^{+}$} & $R^{2}$ & 0.001 & -0.02 & -0.001 & 0.003 \\
\hline & Slope & -1.53 & -0.66 & -8.08 & 1.45 \\
\hline & $\mathrm{p}$ & 0.58 & 0,84 & 0.33 & 0.58 \\
\hline \multirow[t]{3}{*}{$\mathrm{HCO}_{3}^{-}$} & $R^{2}$ & 0.84 & 0.90 & 0.49 & 0.24 \\
\hline & Slope & 1.26 & 2.18 & 2.20 & 1.14 \\
\hline & $p$ & 0.001 & 0.001 & 0.001 & 0.001 \\
\hline \multirow[t]{3}{*}{$\mathrm{Cl}^{-}$} & $R^{2}$ & 0.30 & 0.32 & 0.21 & 0.20 \\
\hline & Slope & -1.28 & -1.45 & -1.24 & -1.37 \\
\hline & $p$ & 0.001 & 0.001 & 0.04 & 0.001 \\
\hline \multirow[t]{3}{*}{ Anion Gap } & $R^{2}$ & 0.20 & 0.11 & 0.08 & 0.04 \\
\hline & Slope & -0.71 & -1.39 & -0.82 & -0.67 \\
\hline & $p$ & 0.006 & 0.01 & 0.23 & 0.03 \\
\hline \multirow[t]{3}{*}{ Lactate } & $R^{2}$ & 0.10 & 0.02 & 0.01 & 0.01 \\
\hline & Slope & -5.54 & -3.42 & -0.95 & 1.58 \\
\hline & $p$ & 0.06 & 0.30 & 0.65 & 0.25 \\
\hline
\end{tabular}


Table 4 Cardiovascular drugs in groups of patients according to ABG analysis values and in the entire cohort

\begin{tabular}{lllll}
\hline & $\begin{array}{l}\text { Mixed respiratory acidosis - } \\
\text { metabolic alkalosis }(N=36)\end{array}$ & $\begin{array}{l}\text { Compensated respiratory } \\
\text { acidosis }(N=51)\end{array}$ & $\begin{array}{l}\text { Mixed respiratory - metabolic } \\
\text { acidosis }(N=23)\end{array}$ & $\begin{array}{l}\text { Hypercapnic COPD, } \\
\text { entire cohort }(N=110)\end{array}$ \\
\hline Diuretics & $33 / 36$ & $40 / 51$ & $22 / 23$ & $95 / 110$ \\
ACE inhibitors & $9 / 36$ & $14 / 50$ & $3 / 20$ & $26 / 106$ \\
Angiotensin receptor antagonists & $12 / 36$ & $15 / 50$ & $4 / 20$ & $31 / 106$ \\
Calcium channel blocking agents & $5 / 36$ & $15 / 50$ & $3 / 20$ & $23 / 106$ \\
Beta-blockers & $3 / 36$ & $4 / 50$ & $0 / 20$ & $7 / 106$ \\
Nitroglycerin & $7 / 36$ & $3 / 50$ & $1 / 20$ & $11 / 106$ \\
Potassium sparing agents & $4 / 36$ & $3 / 50$ & $1 / 21$ & $8 / 107$ \\
\hline
\end{tabular}

alkalosis. In our patients, the use of diuretics and steroids and a relative reduction in circulating blood volume were the main causes of metabolic alkalosis. We observed that patient belonging to group B (respiratory acidosis + metabolic alkalosis) had a better prognosis than patients in group A (compensated respiratory acidosis) with a significantly lower need of NIV.

The admission to ICU was not significantly different in comparison to patients belonging to group $\mathrm{A}$.

These data are conflicting with Terzano et al. who observed that metabolic alkalosis worsened the prognosis of respiratory acidosis in hypercapnic AECOPD patients. In their study, patients with mixed disorder (respiratory acidosis plus metabolic alkalosis) had a longer and higher incidence of NIV support than patients with compensated respiratory acidosis [12].

To explain such different finding, we can speculate that patients with hypercapnic AECOPD managed in an Internal Medicine ward of a General Hospital are quite different from patients managed in a Pulmonary Department of a Teaching Hospital. The latter have scheduled follow up visits and are hospitalized only when the clinical status progressively deteriorates. In contrast, hypercapnic AECOPD patients admitted to an Internal Medicine ward of a General Hospital usually come from the Emergency Department, where they are hospitalized because their clinical status worsened for sudden events (such as diarrhoea, vomiting, fever, sweating, decreased fluid intake) with additional autonomous metabolic alkalosis. Therefore, a diuretic and/or glucocorticoid induced metabolic alkalosis with hypovolemia is often detectable in these patients. These events can be easily managed by blood volume and serum chloride restoring (with saline solution) allowing the clinical improvement and avoiding the NIV support.

In an earlier study, it was shown that in COPD patients with peripheral edema treated with loop diuretics, furosemide withdrawing led to increased ventilation and an improvement in the respiratory acidosis [13].

Lactate concentration was higher in NIV+ group. Our result confirms previous studies in which blood lactate was considered a diagnostic hallmark of tissue hypoxia and respiratory muscle fatigue and, indirectly, of COPD severity in patients not requiring NIV [12]. However, hyperlactataemia (lactate values $>2 \mathrm{mmol} / \mathrm{L}$ ), plays a different role in the three groups of $A B$ disorders. In fact, in group B it was present only in patients not requiring NIV (33\%); in group A was present both in patients requiring (25\%) and not requiring NIV (30\%); and in the group $\mathrm{C}$ it was present in $50 \%$ of patients requiring NIV. In group $B$ the lactate overproduction may reflect a positive response to the stress enabling an alternative energetic way through a carbon units transport between cells ("good lactate"); whereas in group C the lactate hyperproduction is probably due to hypoperfusion ("bad lactate") and should be immediately recognized and treated $[14,15]$. In the patients of the NIV-treated respiratory acidosis group we didn't observe metabolic "post-hypercapnic alcalosis" since the concomitant metabolic component was simultaneously treated and corrected.

We acknowledge that many of the cross-associations between $\mathrm{pCO}_{2}$ and serum electrolytes found in our study are expected, as the $\mathrm{AB}$ balance is closely related to the HE balance. Nevertheless, some remarks seem worthy of focus.

In Brackett's classic experiment, on healthy volunteers, acute hypercapnia was experimentally induced by having them hyperventilating by breathing $\mathrm{CO}_{2}$-enriched (at $7 \%$ and at $10 \%$ ) air. In these subjects, a variation of both $\mathrm{pH}$ and bicarbonates occurred, with a very slight, but significant, increase in $\mathrm{Na}^{+}$and $\mathrm{K}^{+}$, and no changes in $\mathrm{Cl}^{-}$, indeterminate anions (anion gap: $\mathrm{AG}$ ) and lactates concentrations [16]. In a following study with patients with alveolar hypoventilation and absence of clinical conditions potentially responsible for metabolic alkalosis, the response to the chronic hypercapnia was investigated. In this group a decrease in $\mathrm{pH}$ with a progressive increase in $\mathrm{pCO}_{2}$ was seen; moreover, when $\mathrm{pCO}_{2}$ values increased, $\mathrm{Cl}^{-}$decreased whereas no significant association was found with $\mathrm{HE}$ balance $\left(\mathrm{Na}^{+}, \mathrm{K}^{+}\right.$and $\left.\mathrm{AG}\right)$ [17].

In our study population composed by patients with hypercapnic AECOPD, we found a significant association 
between increase in $\mathrm{pCO}_{2}$ and bicarbonate (which reflects the renal compensation) and a statistically significant relationship between the $\mathrm{pCO}_{2}$ increase and serum chloride decrease. Such associations were present in all the subjects studied and was maintained also when dividing the subjects in the 3 subgroups classified according to the ABG disorder. Serum chloride decrease may be related to bicarbonate increase as an attempt to assure the electro-neutrality. We can interpret the data of serum chloride reduction also in the light of the Strong Ion Difference (SID) of Stewart's theory (Stewart). In this case, in hypercapnic AECOPD, after the pulmonary response to increasing $\mathrm{pCO}_{2}$, the renal response starts, by regulating $\mathrm{Na}^{+}$and $\mathrm{Cl}^{-}$, which are the major SID determinants. A relative (compared to $\mathrm{Na}^{+}$concentration) hypochloremia would lead to a SID increase and would indicate a metabolic alkalosis with a bicarbonate increase. According to this theory, therefore, bicarbonate increase reflects a shift in bicarbonate equilibrium of the ratio $\mathrm{H}^{+/} \mathrm{HCO}^{-}$connected with the SID variation [18]. In fact, in the overall group and in the subgroups with compensated respiratory acidosis and respiratory acidosis + metabolic alkalosis, an inverse association between $\mathrm{pCO}_{2}$ and indeterminate anions was found.

\section{Conclusions}

In conclusion our study, performed in a population of inpatients admitted to an Internal Medicine Division for hypercapnic AECOPD, shows that:

1. The AB balance and the HE balance, in agreement with previous studies, are closely interconnected;

2. The electrolytic changes are usually in a range that could be easily corrected with the conventional pharmacological therapy;

3. In hypercapnic respiratory acidosis due to AECOPD, differently from previous studies, the metabolic alkalosis is not a negative prognostic factor neither determines greater NIV support need;

4. The metabolic acidosis in addition to respiratory acidosis is an unfavourable element, since it determines an increased need of NIV and invasive mechanical ventilation support.

\section{Competing interests}

The authors declare that they have no competing interests.

\section{Authors' contributions}

Al and FP drafted the manuscript. MP participated in the design of the study and performed the statistical analysis. AS and MR conceived of the study, and participated in its design and coordination and helped to draft the manuscript. All authors read and approved the final manuscript.

\section{Acknowledgements}

We acknowledge all the staff of Department of Internal Medicine of Cava de' Tirreni Hospital for contribution towards the study by making acquisition of data.

\section{Author details}

'Division of Internal Medicine, Cava de' Tirreni Hospital, University of Salerno, Cava de' Tirreni, SA, Italy. ${ }^{2}$ Division of Pulmonary Medicine, Scafati Hospital, Salerno, Italy. ${ }^{3}$ Division of Internal Medicine, A. Cardarelli Hospital, Naples, Italy. ${ }^{4}$ Division of Pulmonary and Critical Care Medicine, Department of Medicine, Brigham and Women's Hospital, Harvard Medical School, Boston, MA, USA.

Received: 11 March 2016 Accepted: 29 April 2016

Published online: 25 May 2016

\section{References}

1. Wooten EW. Quantitative acid-base physiology using the Stewart model. Crit Care. 2004;8:448-52.

2. Adrogué HJ, Gennari FJ, Galla JH, Madias NE. Assessing acid-base disorders. Kidney Int. 2009;76:1239-47.

3. Terzano C, Conti V, Di Stefano F, Petroianni A, Ceccarelli D, et al. Comorbidity, hospitalization and mortality in COPD: results from a longitudinal study. Lung. 2010;188:321-9.

4. Vestbo J, Hurd SS, Agustí AG, Jones PW, Vogelmeier C, Anzueto A, et al. Global strategy for the diagnosis, management, and prevention of chronic obstructive pulmonary disease: GOLD executive summary. Am J Respir Crit Care Med. 2013;187:347-65.

5. Teasdale G, Jennett B. Assessment of coma and impaired consciousness. A practical scale. Lancet. 1974;2:81-4.

6. Stinebaugh BJ, Austin WH. Acid-base balance: common sense approach. Arch Intern Med. 1967;119:182-8.

7. Aaron SD. Management and prevention of exacerbations of COPD. BMJ. 2014;349:95237.

8. Kramer N, Meyer TJ, Meharg J, Cece RD, Hill NS. Randomized, prospective trial of noninvasive positive pressure ventilation in acute respiratory failure. Am J Respir Crit Care Med. 1995;151:1799-806.

9. Confalonieri M, Garuti G, Cattaruzza MS, Osborn JF, Antonelli M, et al. Italian noninvasive positive pressure ventilation (NPPV) study group. A chart of failure risk for noninvasive ventilation in patients with COPD exacerbation. Eur Respir J. 2005;25:348-55.

10. Plant PK, Owen JL, Elliott MW. Early use of non-invasive ventilation for acute exacerbations of chronic obstructive pulmonary disease on general respiratory wards: a multicentre randomised controlled trial. Lancet. 2000;355:1931-5.

11. Ucgun I, Oztuna F, Dagli CE, Yildirim H, Bal C. Relationship of metabolic alkalosis, azotemia and morbidity in patients with chronic obstructive pulmonary disease and hypercapnia. Respiration. 2008;76:270-4.

12. Terzano C, Di Stefano F, Conti V, Di Nicola M, Paone G, et al. Mixed acid-base disorders, hydroelectrolyte imbalance and lactate production in hypercapnic respiratory failure: the role of noninvasive ventilation. PLoS ONE. 2012;7:e35245.

13. Brijker F, Heijdra YF, van den Elshout FJ, Folgering HT. Discontinuation of furosemide decreases $\mathrm{PaCO}_{2}$ in patients with COPD. Chest. 2002;121:377-82.

14. Brackett Jr NC, Cohen JJ, Schwartz WB. Carbon dioxide titration curve of normal man. Effect of increasing degrees of acute hypercapnia on acid-base equilibrium. N Engl J Med. 1965;272:6-12.

15. Mizock BA. The epatosplanchnic area and hyperlactatemia. A tale of two lactates. Crit Care Med. 2001;29:447-9.

16. Schiraldi F, Guiotto G. Equilibrio acido-base. Ossigeno. Fluidi \& elettroliti. 2012. pp 45-53.

17. Brackett NC, Wingo CF, Muren O, Solano JT. Acid-base response to chronic hypercapnia in man. N Engl J Med. 1969;280:124-30.

18. Alfaro V, Torras R, Ibáñez J, Palacios L. A physical-chemical analysis of the acid-base response to chronic obstructive pulmonary disease. Can J Physiol Pharmacol. 1996;74:1229-35. 